\title{
Resistencia a la Compresión de Carillas Cerámicas de Disilicato de Litio Cementadas con Cemento Resinoso Dual y Cemento Resinoso Dual Autoadhesivo en Premolares Maxilares
}

\author{
Compressive Resistance of Lithium Disilicate Porcelain Laminate Veneers Cemented \\ with Dual Resin Cement and Self-adhesive Dual Resin Cement in Upper Premolars
}

\author{
Brando Mellado Alfaro ; Shilla Anchelia Ramirez * \& Eduardo Quea Cahuana*
}

MELLADO, A. B.; ANCHELIA, R. S. \& QUEA, C. E. Resistencia a la compresión de carillas cerámicas de disilicato de litio cementado con cemento resinoso dual y cemento resinoso dual autoadhesivo en premolares maxilares. Int. J. Odontostomat., 9(1):85-89, 2015.

RESUMEN: El objetivo de este trabajo fue determinar el grado de resistencia a la fuerza de compresión de las carillas de cerámica de disilicato de litio cementadas con dos agentes de resina dual en premolares maxilares. En este estudio experimental in vitro se seleccionaron 30 premolares maxilares, los cuales fueron divididos aleatoriamente en dos grupos de 15 dientes cada uno; posteriormente los especímenes fueron preparados para recibir carillas de cerámica de 0,5 $\mathrm{mm}$ de grosor. Las carillas de grupo 1 se cementaron con un cemento resinoso dual (CRD) y las del grupo 2 con un cemento de resina dual autoadhesivo (CRDA). Luego de cementadas fueron puestas a prueba en una máquina de ensayo universal hasta su fractura por fuerzas de compresión $(\mathrm{N})$ a una velocidad de $1 \mathrm{~mm} / \mathrm{min}$. Para la comparación de medias se utilizó la prueba $t$ de Student $(\alpha=0,05)$. Los resultados mostraron diferencias estadísticamente significativas $(p<0,05)$ entre los grupos estudiados. La media de la resistencia a la compresión de las muestras cementadas con cemento resinoso dual y cemento resinoso dual autoadhesivo fue de $677,47 \mathrm{~N}$ y $5500 \mathrm{~N}$, respectivamente. Se determinó que las carillas de cerámica de disilicato de litio cementadas con CRD tienen mayor resistencia a la fuerza de compresión respecto a las cementadas con CRDA.

PALABRAS CLAVE: laminado dental, cemento de resina, fractura mecánica, cementación.

\section{INTRODUCCIÓN}

Las carillas de cerámica constituyen una alternativa conservadora de recubrimiento parcial, que mejora la estética del sector anterior. Esta restauración ha evolucionado durante las últimas décadas y actualmente se sitúa dentro de las restauraciones más comunes.

Las alteraciones de forma o color en los dientes posteriores saludables, representan una de las condiciones clínicas que demandan el uso de una carilla de cerámica muy fina en ese sector.

Este tipo de restauraciones requieren de un agente de cementación, el cual influye en la adhesión $y$ resistencia a las diferentes fuerzas a las que son sometidas.
Los sistemas de cerámica en la actualidad han experimentado grandes cambios llegando a mejorar sus propiedades mecánicas, no obstante de estos avances las carillas cerámicas continúan restringiéndose solo al sector anterior (Addison et al., 2007; Atsu et al., 2006), sin aún un consenso respecto a su uso en el sector posterior. Los sistemas de cerámica, aunque han mejorado (Addison et al.; Atsu et al.; Okumura et al., 2011), muestran limitaciones mecánicas cuando se utiliza en la región posterior por la incapacidad de la cerámica para absorber eficientemente las fuerzas de tensión debido a su bajo grado de deformación plástica, pudiendo resultar en pequeñas grietas, fracturas y defectos que conducen a su fracaso (Kim et al., 2007).

\footnotetext{
Ayudante de Cátedra de la Facultad de Odontología, Cirujano Dentista, Universidad de San Martín de Porres (USMP), Lima, Perú. "Docente de la Facultad de Odontología, Cirujano Dentista, Universidad de San Martín de Porres (USMP), Lima, Perú
} 
Sin embargo, cuando se establecen procedimientos óptimos de cementado, la capa adhesiva absorbe parcialmente las tensiones que actúan sobre el mismo, redistribuyéndolo dentro de la estructura dental, mejorando el comportamiento mecánico de la carilla de cerámica y la preservación de la integridad de la interfaz diente-restauración (Ausiello et al., 2004).

Magne \& Douglas (1999) y Stokes \& Hood (1993) observaron que cuando la carilla se une al sustrato dental reproducen el comportamiento mecánico y estructural de un diente intacto.

El cemento de resina es responsable de la unión del material restaurador indirecto al diente preparado (Kitasako et al., 2001). Su composición es similar a las resinas compuestas, pero en diferentes proporciones (Beloti et al., 2000).

Actualmente, existen cementos resinosos autoadhesivos que por su aplicación directa, sin necesidad de pre tratamiento del sustrato dental, constituyen una opción clínicamente atractiva, sin embargo, para su elección no debe dejar de considerarse sus propiedades físicas y químicas (Duarte et al., 2011). La unión al sustrato ha demostrado la supervivencia clínicamente aceptable de las carillas anteriores hasta un máximo de 10 años que van desde $64 \%$ a $91 \%$, siendo su principal complicación las fracturas de la misma, la cual representa hasta un $3 \%$ del total de cementadas según lo reportado en algunos estudios clínicos (Chun et al., 2010).

Abou-Madina et al. (2012) realizaron un estudio para comparar la influencia de dos diferentes cementos sobre la resistencia a la compresión de coronas posteriores de cerámica de disilicato de litio. Asimismo, Blatz et al. (2008), en un estudio in vitro observaron la resistencia a la fractura de coronas de cerámica de alúmina cementadas con tres tipos de cementos y diferentes técnicas de cementación. Ambos estudios evaluaron la correlación existente entre los cementos y su resistencia a la fractura en las coronas libres de metal.

En la actualidad, al rehabilitar un diente anterior o posterior se busca la preservación del tejido a través de una mínima intervención de tallado en la superficie dental. Las carillas de cerámica en el sector posterior son una opción para devolver la funcionalidad y estética de los dientes en esa zona. Sin embargo, para prever su supervivencia clínica, se debe buscar el material cementante que garantice una unión aceptable y que preserve la interfaz diente-restauración.

\section{MATERIAL Y MÉTODO}

Para este estudio experimental in vitro se recolectaron y limpiaron 30 dientes premolares maxilares recién extraídas, libres de caries e intactos, los mismos fueron divididos aleatoriamente en dos grupos de 15 dientes cada uno (Grupo 1 se cementaron con un cemento resinoso dual - CRD y las del grupo 2 con un cemento de resina dual autoadhesivo - CRDA). Las muestras fueron posicionadas verticalmente en un zócalo de acrílico que cubrió la raíz hasta $2 \mathrm{~mm}$ antes de la unión cemento esmalte. Luego se confeccionaron guías de siliconas a cada premolar para tener un mejor control de la profundidad del desgaste.

Los dientes fueron preparadas en su superficie bucal, siguiendo las dos inclinaciones anatómicas de su superficie, a una profundidad de $0,3 \mathrm{~mm}$ y $0,5 \mathrm{~mm}$ en la mitad gingival y oclusal respectivamente.

En oclusal, se preparó a $0,5 \mathrm{~mm}$ de profundidad y se abarcó la cúspide bucal hasta el surco central; la cara proximal se preparó siguiendo la proyección de las preparaciones de la superficie bucal y superficie oclusal hasta unirlas de forma oblicua con una profundidad de 0,5 mm (Fig. 1).

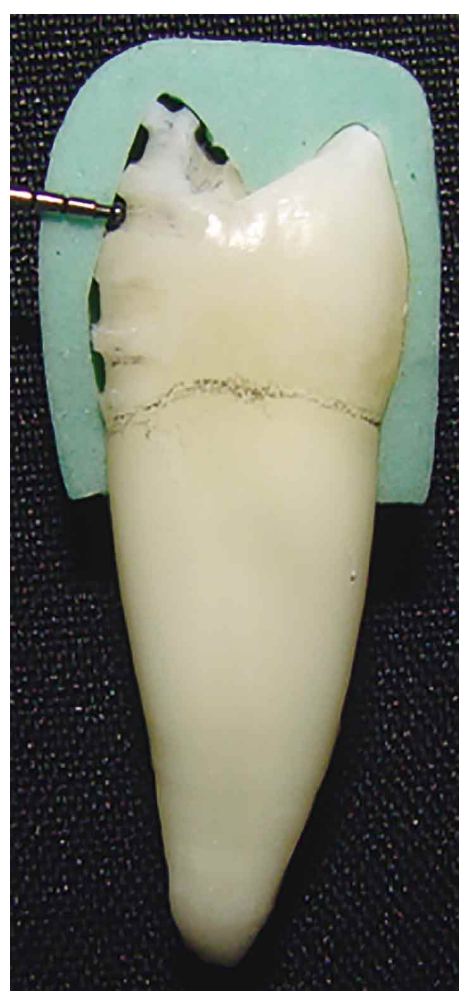

Fig. 1. Control de la profundidad de la preparación. 
Se utilizó silicona por condensación (Zetaplus, Oranwash L, Indurent gel, Zhermack SpA, Roma, Italia) para la toma de impresión empleando la técnica a dos pasos y siguiendo las instrucciones del fabricante. Se hizo el vaciado con yeso tipo IV (elite rock, Zhermack SpA, Roma, Italia). Las carillas cerámicas de disilicato de litio (IPS e. Max press, ivoclar vivadent, Schaan, Liechtenstein) fueron elaborados en el Laboratorio de Prótesis de la Facultad de Odontología de la Universidad de San Martín de Porres.

Las carillas preparadas para el primer grupo se cementaron con un cemento resinoso dual (CRD) (Allcem, FGM, SC, Brazil) y en el segundo grupo con un cemento de resina dual autoadhesivo (CRDA) (Relyx U200, 3M Espe, Neuss, Alemania). Para ambos grupos se procedió con la preparación de las carillas con ácido fluorhídrico al $10 \%$

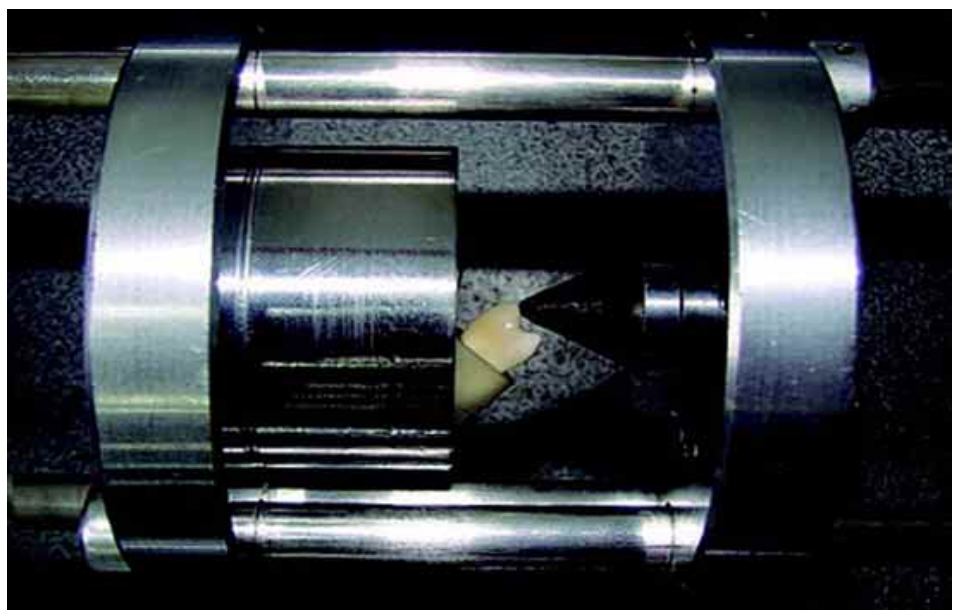

Fig. 2. Muestra sometida a fuerza de compresión en máquina de ensayo universal simulando guía de desoclusión canina.
(Condacporcelana, FGM, SC, Brazil) durante 20 segundos y silano (Prosil, FGM, SC, Brazil). Luego se procedió a la aplicación de los cementos según indicaciones del fabricante.

Posteriormente, las muestras fueron colocadas en una máquina de ensayo universal hasta su lograr su fractura por fuerzas de compresión $(\mathrm{N})$ a una velocidad de $1 \mathrm{~mm} / \mathrm{min}$, con una carga inicial de $150 \mathrm{~N}$ en la parte superior de la cúspide y con una inclinación de $45^{\circ}$ con el eje longitudinal del diente, simulando la guía de desoclusión canina en función de grupo parcial o completo (Fig. 2), posteriormente la carga fue aumentando hasta conseguir la fractura de la carilla.

Para el análisis estadístico se utilizó la prueba t de Student para muestras independientes $(\alpha=0,05)$.

\section{RESULTADOS}

Todas las muestras superaron los valores normales (150 N) sin presentar daño visible en las carillas (Fig. 3). Por lo que fueron sometidas a una mayor carga hasta lograr la fractura a fin de determinar la fuerza máxima de resistencia de cada grupo de cemento (Tabla I).

En la prueba $\mathrm{T}$, se observa que el valor del estadístico fue 2,511 (con 28 grados de libertad) y valor "p" asociado es de 0,018 $(p<0,05)$, demostrando la diferencia significativa entre los dos tipos de cemento utilizados (Fig. 4).

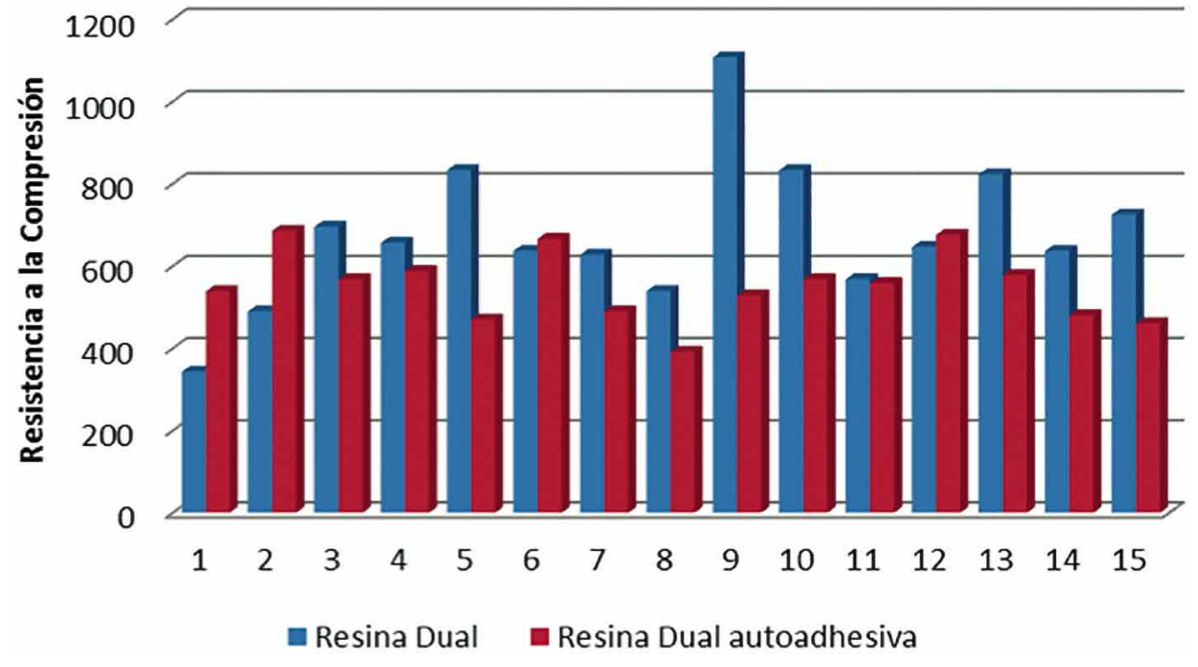

Fig. 3. Valores obtenidos de cada muestra en el momento de la fractura. 
Tabla I. Promedio de los valores de ambos grupos de estudio.

\begin{tabular}{lcccc}
\hline Grupo & Máximo (N) & Mínimo (N) & Media (N) & DE \\
\hline Cemento resinoso dual & 1107 & 343 & 677,47 & 177,683 \\
Cemento resinoso dual autoadhesivo & 686 & 392 & 550,00 & 84,148 \\
\hline
\end{tabular}

$P$ valor $=0,018(<0,05)$.

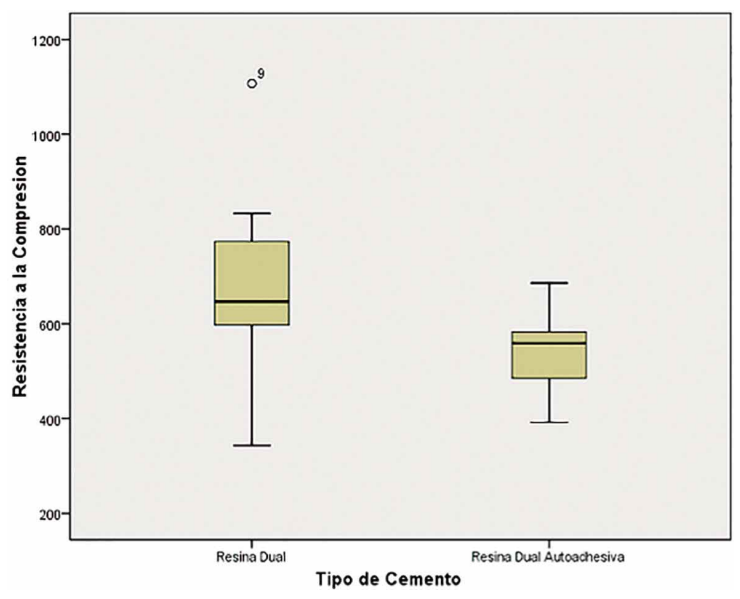

Fig. 4. Diagrama Box-Plot de la resistencia a la compresión establecida por el tipo de cemento.

\section{DISCUSIÓN}

El cemento de resina debe ser capaz de absorber las fuerzas de compresión y la reducción de cargas atípicas sobre la carilla de cerámica, con el fin de mantener la estabilidad del complejo diente-restauración. Tomando en cuenta estos indicadores se puso a prueba las carillas de cerámica de disilicato de litio. La resistencia a la compresión de las carillas cementadas con cemento resinoso dual (CRD) y cemento resinoso dual autoadhesivo (CRDA) superaron los valores de la fuerza de compresión que se produce durante los movimientos de lateralidad en función de grupo total o parcial: $150 \mathrm{~N}$ (Okeson, 1993).

Los valores obtenidos evidencian una diferencia entre ambos cementos ante la fuerza de compresión aplicada en las carillas, coincidiendo con lo reportado por Blatz et al., quienes compararon la influencia de 3 materiales de cementación en la resistencia a la fractura en coronas de alúmina, para ello emplearon: fosfato de zinc (grupo ZOP), cemento resinoso dual autoadhesivo (HYB) y cemento resinoso dual autograbante (ADH), encontrando diferencia significativa entre los sistemas de cementación.

No obstante nuestros resultados discrepan con los obtenidos por Abou-Madina et al., cuyo propósito fue evaluar la efectividad de diferentes cementos adhesivos (cemento resinoso dual autograbante y cemento resinoso dual autoadhesivo, con y sin termociclado) en la resistencia a la fractura de coronas posteriores de disilicato de litio; los resultados de resistencia a la fractura que obtuvieron no fueron afectados significativamente.

Sin embargo, la media obtenida en los estudios mencionados, difieren mucho de los nuestros, esto podría deberse a que utilizaron coronas y no carillas, además las fuerzas fueron dirigidas de formas distintas sobre las coronas; Blatz et al., utilizaron un simulador de masticación y Abou-Madina et al. aplicaron fuerzas en sentido vertical, por otro lado el espesor de las restauraciones y el área de soporte de las coronas eran mayores.

Ciertos factores pueden influir en la resistencia a la compresión de un determinado material, como la preparación dentaria que debe evitar las zonas críticas y frágiles, un espesor mínimo requerido y homogéneo de la restauración, el agente cementante que asienta la restauración sin dejar espacios vacíos consideradas áreas débiles muy susceptibles a la fractura, y la capa de adhesivo. Blatz et al., al comparar el cemento resinoso autograbante con el cemento autoadhesivo llegaron a la conclusión que la técnica de cementación afecta significativamente la resistencia a la fractura. En nuestro estudio comparamos el cemento resinoso dual con un cemento resinoso autodhesivo, observándose que el cemento resinoso dual presentó mayor resistencia a la fuerza de compresión, coincidiendo con lo reportado por Blatz et al. Por ende los protocolos de cementación en los cuales se realice una preparación previa del sustrato con adhesivo tendrían una mayor resistencia a la compresión que aquellos en los cuales no se realice una preparación previa del sustrato con adhesivo.

En ambos grupos estudiados en nuestra investigación los valores mínimos de la resistencia a la compresión fueron mayores a la fuerza fisiológicas generadas durante los movimientos de lateralidad en función de grupo total o parcial, se encontró además diferencia significativa entre las carillas cerámicas de disilicato de litio cementadas con CRD y CRDA obteniéndose un valor de $p=0,018$,

CONCLUSIONES. Las carillas de cerámica de disilicato de litio cementadas con cemento resinoso dual tienen mayor resistencia a la fuerza de compresión que las cementadas con cemento resinoso dual autoadhesivo. 
MELLADO, A. B.; ANCHELIA, R. S. \& QUEA, C. E. Compressive resistance of lithium disilicate porcelain laminate veneers cemented with dual resin cement and self-adhesive dual resin cement in upper premolars. Int. J. Odontostomat., 9(1):8589, 2015.

ABSTRACT: The aim of this work was to establish the resistance degree to the compression force in porcelain laminate. In this experimental in vitro study 30 premolars were selected and randomly divided in groups of 15 teeth each, subsequently specimens were prepared for ceramic veneers $0.5 \mathrm{~mm}$ thick. The first group was cemented with dual resin cement (CRD) and the second with dual adhesive resin cement (CRDA). After cementation was tested in a universal test machine until fracture compression forces occurred $(\mathrm{N})$ at $1 \mathrm{~mm} / \mathrm{min}$ speed. Student's t-test $(a=0.05)$ was used to compare means. The results showed statistically significant differences $(p<0.05)$ between groups. The mean compressive strength for cemented samples was $677.47 \mathrm{~N}$ and $550.0 \mathrm{~N}$ for dual resin cement and auto-adhesive dual resin cement respectively. It was found that porcelain laminate veneers made of lithium disilicate and cemented with CRD have greater resistance to compression force compered to CRDA.

KEY WORDS: dental veneers, resin cement, mechanical fracture, cementation.

\section{REFERENCIAS BIBLIOGRÁFICAS}

Abou-Madina, M. M.; Özcan, M. \& Abdelaziz, K. M. Influence of resin cements and aging on the fracture resistance of IPS e.max press posterior crowns. Int. J. Prosthodont., 25(1):335, 2012.

Addison, O.; Marquis, P. M. \& Fleming, G. J. The impact of modifying alumina air abrasion parameters on the fracture strength of a porcelain laminate restorative material. Dent. Mater., 23(11):1332-41, 2007.

Archangelo, C. M.; Rocha, E. P.; Anchieta, R. B.; Martin, M. Jr.; Freitas, A. C. Jr.; Ko, C. C. \& Cattaneo, P. M. Influence of buccal cusp reduction when using porcelain laminate veneers in premolars. A comparative study using 3-D finite element analysis. J. Prosthodont. Res., 55(4):221-7, 2011.

Atsu, S. S.; Kilicarslan, M. A.; Kucukesmen, H. C. \& Aka, P. S. Effect of zirconium-oxide ceramic surface treatments on the bond strength to adhesive resin. J. Prosthet. Dent., 95(6):4306, 2006.

Ausiello, P.; Rengo, S.; Davidson, C. L. \& Watts, D. C. Stress distributions in adhesively cemented ceramic and resincomposite Class II inlay restorations: a 3D-FEA study. Dent. Mater., 20(9):862-72, 2004.

Beloti, A. M.; Varjão, F. M.; Segalla, J. C. M. \& Andrade, L. E. H. Avaliação da espessura de película de cimentos resinosos. $J$. Bras. Odontol. Clín., 4:33-6, 2000.

Blatz, M. B.; Oppes, S.; Chiche, G.; Holst, S. \& Sadan, A. Influence of cementation technique on fracture strength and leakage of alumina all-ceramic crowns after cyclic loading. Quintessence Int., 39(1):23-32, 2008.

Chun, Y. H.; Raffelt, C.; Pfeiffer, H.; Bizhang, M.; Saul, G.; Blunck, U. \& Roulet, J. F. Restoring strength of incisors with veneers and full ceramic crowns. J. Adhes. Dent., 12(1):45-54, 2010.

Duarte, S. Jr.; Sartori, N.; Sadan, A. \& Phark, J. H. Adhesive Resin Cements for Bonding Esthetic Restorations: A Review. Quintessence Dent. Technol., 34:40-66, 2011.
Kim, B.; Zhang, Y.; Pines, M. \& Thompson, V. P. Fracture of porcelain-veneered structures in fatigue. J. Dent. Res., 86(2):142-6, 2007.

Kitasako, Y.; Burrow, M. F.; Katahira, N.; Nikaido, T. \& Tagami, J. Shear bond strengths of three resin cements to dentine over 3 years in vitro. J. Dent., 29(2):139-44, 2001.

Magne, P. \& Douglas, W. H. Additive contour of porcelain veneers: a key element in enamel preservation, adhesion, and esthetics for aging dentition. J. Adhes. Dent., 1(1):81-92, 1999.

Okeson, J. P. Management of temporomandibular disorders and occlusion. 6th ed. St. Louis, Mosby-Year Book; 1993.

Okumura, N.; Stegaroiu, R.; Nishiyama, H.; Kurokawa, K.; Kitamura, E.; Hayashi, T. \& Nomura, S. Finite element analysis of implant-embedded maxilla model from CT data: comparison with the conventional model. J. Prosthodont. Res., 55(1):2431, 2011.

Stokes, A. N. \& Hood, J. A. Impact fracture characteristics of intact and crowned human central incisors. J. Oral Rehabil., 20(1):8995, 1993

Dirección para Correspondencia:

Brando Mellado Alfaro

Facultad de Odontología

Universidad de San Martín de Porres (USMP)

Lima

PERÚ

Email: jeffer33@hotmail.com

Recibido : 05-11-2014

Aceptado: 04-03-2015 